\title{
Detection of Symmetries in Cattle Skulls
}

\author{
P.M. Parés-Casanova ${ }^{1, *}$, B.G. Gambo ${ }^{2}$, A. Yahaya ${ }^{2}$ and J.O. Olopade ${ }^{3}$ \\ ${ }^{1}$ Department of Animal Science, University of Lleida, Catalonia, Spain; ${ }^{2}$ Department of Veterinary Anatomy, \\ University of Maiduguri, Nigeria; ${ }^{3}$ Department of Veterinary Anatomy, University of Ibadan, Nigeria
}

\begin{abstract}
A sample of adult individuals from two ethnological groups ("European" $n=36$ and "African" $n=10$ ) that exhibited a normal craniofacial phenotype on their dorsal aspect was studied by means of geometric morphometric methods. Eleven landmarks were selected, corresponding to the neurocranium and viscerocranium. Both fluctuating as directional asymmetries were detected. The latter accounted for the largest proportion of the total variation, causing the main variation in symmetric shape, the former being higher in Kuri cattle. Detected fluctuating asymmetries could indicate an overall poor quality or general low health condition of individuals, due to the harder conditions (climatic, tropical) in which Kuri breed must thrive. Detected directional asymmetry, on the other side, could reflect just a mere functional lateralization of Bos taurus, as it has been established for other domestic animals.
\end{abstract}

Keywords: Bos taurus, Cranial Morphology, Directional Asymmetry, Fluctuant Asymmetry, Geometric Morphometrics.

\section{INTRODUCTION}

Taurine cattle (Bos taurus taurus) is subspecies of domesticated cattle from east, and are descendants of the aurochs [1]. These subspecies were originally considered to be distinct species, but are now grouped alongside indicus (zebu) and aurochs as one species, Bos taurus [2].

Fluctuating asymmetry (FA) concerns a particular form of biological asymmetry, in which there appear small, randomly directed deviations from perfect symmetry [3]. Directional asymmetry (DA) is characterized by asymmetry distribution that is not centered around zero but is biased significantly, towards larger traits either on the left or the right side [3]. Antisymmetry (AS) is characterized by being centered around a mean of zero; however, symmetric individuals are rarer than those seen in FA distributions, such that the distribution is platykurtic or, in the extreme, bimodal [3]. Although a great many studies have revealed FA, some have also revealed subtle DA. The value of these alternative patterns of bilateral variation as measures of developmental precision, however, remains open to debate. In this paper, we apply geometric morphometrics to study bilateral symmetries in cattle skulls.

\section{MATERIALS AND METHODS}

\section{Sample Composition}

The sample was composed of adult individuals from two ethnological groups ("European" $n=36$ and

*Address correspondence to this author at the Department of Animal Science, University of Lleida, Catalonia, Spain; Tel: 34973706460;

E-mail: peremiquelp@ca.udl.cat
"African" $n=10$ ) that exhibited a normal craniofacial phenotype. No differences between sexes (for most of the specimens it was unknown) were considered. A subset of 6 individuals of Greek origin was referred to as the "Greek" subset. These skulls were from the Faculty of Animal Science and Aquaculture, Agricultural University in Athens (Greece). The rest of European skulls are deposited at the Department of Animal Science in the University of Lleida. "African" group was composed exclusively of Kuri cattle. These skulls were from Gambo's collection, from the Department of Veterinary Anatomy, University of Maiduguri, Nigeria. The rest of European specimens were subclustered disregarding their origin.

"Greek" specimens belonged to longhorn's types. Kuri breed is from lake Chad belonging to West African Humpless Longhorns group [1]. It is a very tall taurine $(135 \mathrm{~cm}$ at the withers), heavy-boned with gigantic horns, sometimes described as "bony" horns, which are light and porous [1]. A note on this African breed: increased anthropogenic pressure on the environment leading to habitat loss, as is the case for the Chad lake area, requires fast and precise ethnodiversity assessments and therefore solid morphologic knowledge. Unfortunately, representative collections, as well as skilled specialists, are still needed. Proper analytical tools can provide the means to compensate for this shortage by assisting non-specialists researchers, such as biologists and anthropologists, to identify breeds and also by assisting systematists in alpha taxonomic work, using collection specimens, in the analysis and interpretation of morphological differences and in delimiting morphological breed boundaries. In this sense, the study of skull 
morphology is an essential step toward this goal because the skull contains most diagnostic characters.

\section{Obtention of Images}

The use of geometric morphometrics allows a metric partition of the form of an object into components of size and shape, but it also uses landmarks as form descriptors, therefore making subsequent comparisons homologous [4]. Each skull was photographed in a dorsal view using a digital camera. Eleven landmarks (LM) were selected, corresponding to the neurocranium (4 LM) and viscerocranium (4 LM), the rest corresponding to sagittal plane (occipital protuberance, frontal spine and most rostral point of incisive bone) (Figure 1). In addition to being highly repeatable; these landmarks encompass elements of the entire cranium. The $x$ and
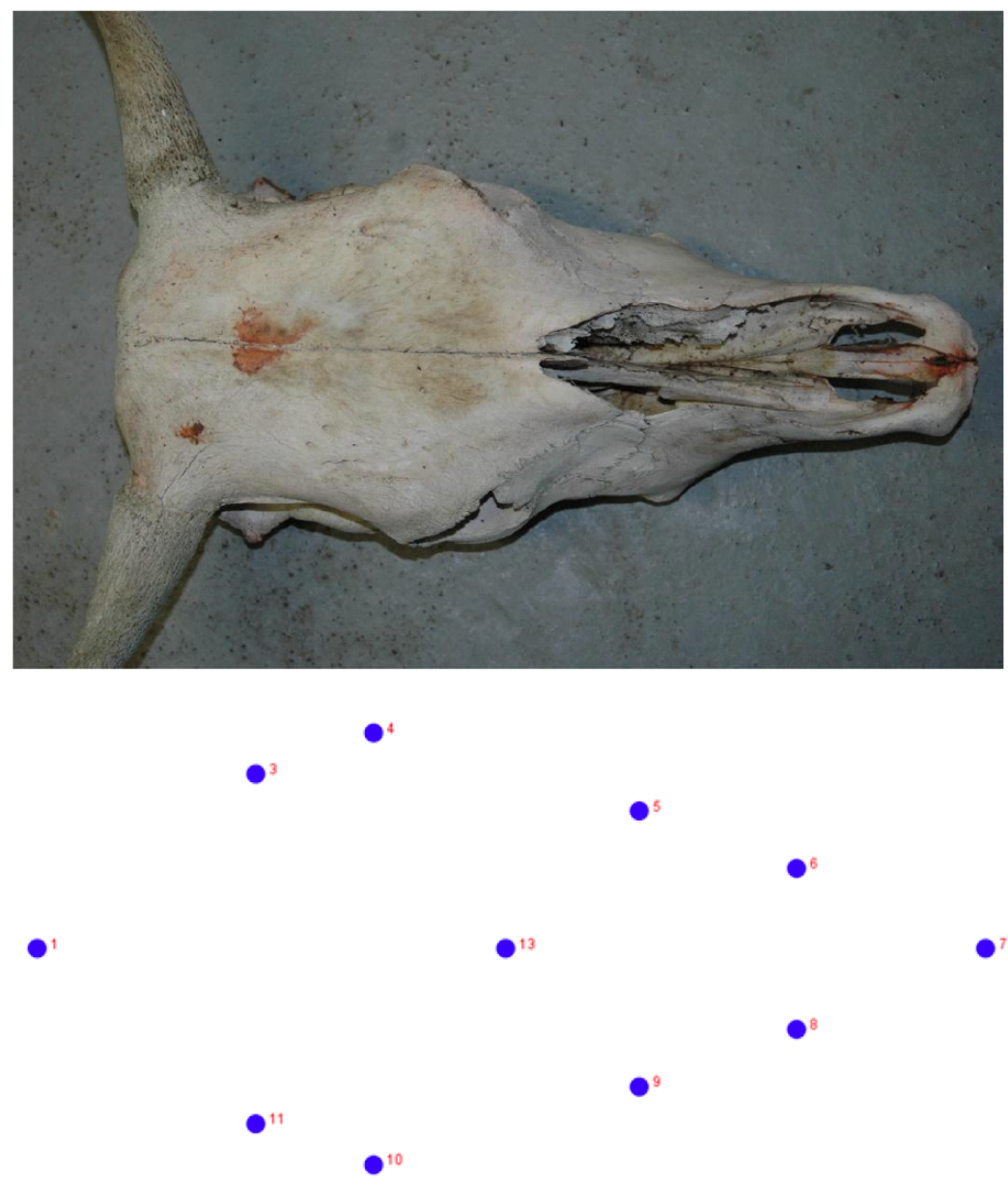

Figure 1: Dorsal view of the skull (rostral at right). Eleven landmarks (LM) were selected, corresponding to the neurocranium (4 LM) and viscerocranium (4 LM), the rest corresponding to saggital plane (occipital protuberance, frontal spine and most rostral point of incisive bone).

1. Occipital protuberance; LM placed at the midline of the most caudal border of the dorsal surface of the skull.

3. Left temporal line; LM placed at the middle of the left temporal line.

4. Left zygomatic process of frontal bone; LM placed on the most lateral aspect of the left zygomatic process of frontal bone.

5. Left Facial tuber; LM placed on the lateral edge of the left facial tuber.

6. Left nasoincisive suture; LM placed on the lateral end of left nasoincisive suture as viewed dorsally.

7. Rostral end of the interincisive suture; LM placed at the most rostral end of the interincisive suture.

8. Right nasoincisive suture; LM placed on the lateral end of right nasoincisive suture as viewed dorsally.

9. Right Facial tuber; LM placed on the lateral edge of the right facial tuber.

10. Right zygomatic process of frontal bone; LM placed on the most lateral aspect of the right zygomatic process of frontal bone.

11. Right temporal line; LM placed at the middle of the right temporal line.

13. Frontonasal suture; LM placed at the midline of frontonasal suture. 
$y$ coordinates of each LM were digitized using tpsDig v. 2.26 [5]. This procedure was repeated twice by one of the authors (BG). The resulting coordinates were subjected to a generalized Procrustes analysis, which removes all the information unrelated to shape, with MorhoJ v.1.06d [6].

\section{Obtention of Procrustes Coordinates}

Landmark configurations were submitted to a Procrustes superimposition when centroid sizes are computed and all information on a position, orientation and isometric size of landmark configurations are removed, resulting in sets of aligned or Procrustes coordinates, which contains only shape information unrelated to isometric [7].

\section{Study of Symmetry}

An alternative approach for quantifying the different components of variation, which is Procrustes ANOVA [8], was also used. The ANOVA approach was originally developed for linear measurements of bilaterally symmetrical structures and was a two-factor, mixed-model ANOVA design containing individuals and sides as the factors [9]. DA ("sides", one side is systematically different from the other one), FA ("individual $x$ side interaction", small random deviations from perfect symmetry), and their respective error were included as effects. In summary, the magnitude of mandible asymmetry for each respondent was measured following three steps. In the first step, reflected copies of each landmark were generated by reversing the side of its $\mathrm{x}$-coordinate. Then, the Procrustes average of each mandible was defined as the middle of the line passing between the original landmark and the reflected copy of the corresponding landmark. The new mandible shape created by connecting these average landmarks was perfectly symmetrical. Lastly, the asymmetry of each skull was calculated as the difference between the original and the mirror configurations or, equivalently, the landmark deviations of the original configuration from the average landmarks. Our configuration protocol considered 4 paired landmarks to estimate the level of asymmetry (e.g., 2-10, 3-9, 4-8 and 5-7). The assumption of isotropic variation at each landmark was questionable because the scatters of landmark positions around the overall consensus were not circular. Consequently, MANOVA tests were carried out. Since it avoids the assumption of equal and isotropic variation at each landmark and thus takes into account the structure of shape variation, the MANOVA test has a higher statistical power. The statistical significance of the relationship between the shape scores and the centroid sizes was assessed by a permutation test with 10,000 rounds of random permutations [10].

\section{Statistical Procedures}

For analysis of a potential FA data set, certain criteria must be met: the measurements must represent actual deviations from symmetry and not measurement error, and the distribution of fluctuating asymmetry must conform to that expected for it, rather than for DA or AS [3]. The procedures we used is a two-factor, mixed model analysis of variance (ANOVA), which determined whether FA is significantly different from measurement error. This ANOVA design contains individuals and sides as the factors. DA ("sides", one side is systematically different from the other), FA ("individual $x$ side interaction", small random deviations from perfect symmetry), and their respective errors are included as effects. Once asymmetry has been established to exist in a trait, data analysis should proceed by testing for the relation between trait size and asymmetry. If there is a relationship, this needs to be accounted for, and a Canonical Variate analysis (CVA) was applied for controlling for size this dependence. Shape asymmetry components (shape differences between sides) were considered for this final analysis and $p$-values were obtained from permutation tests $(10,000$ permutation rounds) for Mahalanobis distances among 3 sub-sets.

\section{RESULTS}

Since the amount of both FA and DA greatly exceeded that of measurement error, the test for the sides yielded a highly significant result (Table 1), being both asymmetries statistically significant $(p<0.01)$. DA accounted for the largest proportion of the total variation, causing the main variation in symmetric shape. FA was higher in Kuri cattle. Individual effect represents the variation between individuals. "Side" quantifies directional asymmetry. "Ind x Side" quantifies fluctuating asymmetry. "Error" quantifies the residual variation due to measurement error. In CVA for asymmetry components, CV1 appeared to account a $79.16 \%$ of the total observed variance and it allowed the statistically significant difference between Kuri cattle to the others $(p<0.001)$. The right side of cranium tended to be smaller than left one. Regression on centroid size (logarithmically transformed) showed that only a $3.35 \%$ of asymmetry was explained by size changes. 
Table 1: Results of Two-Way ANOVA for Shape. Since the amount of DA ("side") and FA ("Ind * Side") greatly exceeded that of measurement error, the test for the sides yielded a highly significant. DA accounted for the largest proportion of the total variation, causing the main variation in symmetric shape. The table contains the Procrustes sums of squares (SS), Procrustes mean squares (MS), degrees of freedom (df), Goodall's F statistic (F) and the associated parametric p-value.

\begin{tabular}{|c|c|c|c|c|c|}
\hline Effect & SS & MS & Df & $\boldsymbol{F}$ & $\boldsymbol{p}$-value \\
\hline \hline Individual & 0.031030 & 0.001724 & 18 & 6.55 & 0.0001 \\
\hline Side & 0.012983 & 0.001443 & 9 & 5.48 & 0.0011 \\
\hline Ind * Side & 0.004734 & 0.000263 & 18 & 11.02 & 0.0001 \\
\hline Error & 0.001289 & $2.39 \mathrm{E}-05$ & 54 & & 1 \\
\hline Residual & 0.171667 & 0.000111 & 1548 & & \\
\hline
\end{tabular}

\section{DISCUSSION}

FA reflects developmental instability, e.g. individual's inability to develop the intended phenotype under given environmental conditions. If a growing individual fails to cope with perturbing factors, the development of such traits may be disrupted, resulting in non-identical development on both sides of the plane of symmetry [11] and so appearing FA. Among populations, larger values of FA have been interpreted to reflect worse environmental conditions and hence decreased welfare [11]. Within populations, larger values of FA were interpreted to reflect lower individual quality in terms of fitness or productivity. The idea behind this concept is that individuals of low quality cannot control their development precisely, and consequently more often develop different phenotypes on both sides [12]. To interpret the higher level of FA in Kuri cattle could clearly indicate an overall poor quality or general low health condition of individuals, due to the harder conditions (climatic, tropical) in which this breed must thrive.

Detected DA, on the other side, could reflect just a mere functional lateralization of bovines, as it has been established for other domestic animals [11, 13].

\section{REFERENCES}

[1] Porter V, Cattle. A Handbook to the Breeds of the World. Ramsbury, Marlborough: Christipopher Helm Ltd, 1991.

[2] Porter V, Alderson L, Hall SJG, Sponenberg P. Mason's World Encyclopedia of Livestock Breeds and Breeding. CABI, 2016.

https://doi.org/10.1079/9781845934668.0000

[3] Tomkins JL, Andrews S. Fluctuating Asymmetry. Encycl. Life Sci 2001; 1-5.
[4] Cordeiro-Estrela P, Baylac M, Denys C, Marinho-Filho J. Interspecific Patterns of Skull Variation Between Sympatric Brazilian Vesper Mice: Geometric Morphometrics Assessment. J Mammal 2006; 87(6): 1270-1279. https://doi.org/10.1644/05-MAMM-A-293R3.1

[5] Rohlf FJ. 'tpsDig v. 2.26'. http://life.bio.sunysb.edu/morph/ index.html, 2016.

[6] Klingenberg CP. Morpho J: An integrated software package for geometric morphometrics. Mol Ecol Resour 2011; 11(2): 353-357.

https://doi.org/10.1111/j.1755-0998.2010.02924.x

[7] Astúa D. Morphometrics of the largest New World marsupials, opossums of the genus Didelphia (Didelphimorphia, Didelphidae). Oecologia Aust 2015; 19(1): 117-142.

https://doi.org/10.4257/oeco.2015.1901.08

[8] Klingenberg CP, Mclntyre GS, Zaklan SD. Left-right asymmetry of fly wings and the evolution of body axes. Proc Biol Sci 1998; 265: 1255-1259. https://doi.org/10.1098/rspb.1998.0427

[9] Palmer AR, Strobeck C. Fluctuating Asymmetry Analyses Revisited, in Developmental Instability (DI): Causes and Consequences, Oxford University, Ed. Oxford: Michal Polak, Publisher, 2001.

[10] Savriama Y, Neustupa J, Klingenberg CP. Geometric morphometrics of symmetry and allometry in Micrasterias rotata (Zygnemophyceae, Viridiplantae), Nov. Hedwigia Suppl 2010; 136: 43-54.

[11] Tuyttens FA, Maertens L, Van Poucke E, Van Nuffel A, Debeuckelaere S, Creve J, Lens L. Measuring fluctuating asymmetry in fattening rabbits: a valid indicator of performance and housing quality? J Anim Sci 2005; 83(11): 2645-2652. https://doi.org/10.2527/2005.83112645x

[12] Galeotti P, Sacchi R, Vicario V. Fluctuating asymmetry in body traits increases predation risks: tawny owl selection against asymmetric woodmice. Evol Ecol 2005; 19(4): 405418.

https://doi.org/10.1007/s10682-005-8309-z

[13] Parés-Casanova PM. Existence of mandibular directional asymmetry in the European wild boar (Sus scrofa Linnaeus, 1758). J Morphol Sci 2014; 31(4): 1-5. https://doi.org/10.4322/jms.064613 